Review

International Journal of Biological Sciences

ISSN 1449-2288 www.biolsci.org 2007 3(5):318-327

(C) Ivyspring International Publisher. All rights reserved

\title{
In search of a function for the most frequent naturally-occurring length polymorphism (IIFNLP) of the HIV-I LTR: Retaining functional coupling, of Nei and RBF-2, at RBEIII?
}

\author{
Mario Clemente Estable \\ Department of Chemistry and Biology, Ryerson University, 350 Victoria Street, Toronto, Ontario, Canada, M5B 2K3
}

Correspondence to: Dr. Mario Clemente Estable, mestable@Ryerson.ca

Received: 2007.05.15; Accepted: 2007.06.07; Published: 2007.06.11

Although the prototypical HIV-1 LTR sequences were determined 22 years ago from the initial isolate, elucidating which transcription factors are critical to replication in vivo, has been difficult. One approach has been to examine HIV-1 LTRs that have gone through the gamut of in vivo mutation and selection, in search of absolutely conserved sequences. In this vein, RBEIII sequences are virtually $100 \%$ conserved in naturally occurring HIV-1 LTRs. This is because when they are mutated, the MFNLP recreates an RBEIII site. Here, I enumerate some retroviral mutation mechanisms, which could generate the MFNLP. I then review the literature corresponding to the MFNLP, highlighting the discovery in 1999, that RBEIII and MFNLP sequences, bind USF and TFII-I cooperatively, within the context of earlier and later work that suggests a role in HIV-1 activation, through T-cell receptor engagement and the MAPK cascade. One exception to the nearly absolute conservation of RBEIII, has been a group of long term non progressors (LTNP). These patients harbor deletions to the Nef gene. However, the Nef gene overlaps with the LTR, and the LTNP deletions abrogate RBEIII, in the absence of an MFNLP. I suggest that the MFNLP retains functional coupling between the MAPK-mediated effects of Nef and the HIV-1 LTR, through RBEIII. I propose that difficult-to-revert-mutations, to either Nef or RBEIII, result in the convergent LTNP Nef/LTR deletions recently observed. The potential exploitation of this highly conserved protein-binding site, for chimeric transcription factor repression (CTFR) of HIV-1, functionally striving to emulate the LTNP deletions, is further discussed.

Key words: HIV-1 polymorphisms, MFNLP

\section{HIV-1 polymorphisms}

In the 24 years since the discovery of HIV-1 [1], with the impetus to cure AIDS, there have been an unprecedented number of publications examining retroviral polymorphisms. These have led to significant advances in understanding mechanisms generating them. In particular, when a provirus replicates along with host cellular DNA during mitosis, progeny are extremely homogenous. A case in point is clonal expansion of HTLV-1 [2], resulting in small variability over centuries [2-5]. This can be attributed to the high fidelity of mammalian cellular DNA replication, mediated by the 3'-5' exonuclease proofreading activity of DNA polymerase [6].

When a provirus replicates exogenously by infection, progeny can be extremely heterogeneous $[4,7]$. Since exogenous retroviral replication involves transcription and reverse transcription, both could contribute towards generating polymorphisms. However, RNA polymerase appears to have transcript-assisted proofreading activity [8]. Therefore, for exogenously replicating retroviruses, such as HIV-1, polymorphisms are primarily generated during error prone reverse transcription (Fig. 1) [9, 10].

In particular the mis-incorporations produced by
RNA-templated DNA synthesis and DNA-templated DNA synthesis (Fig. 1), have been proven to be exacerbated by dNTP imbalances both in vitro and in vivo [11-14]. Proof of the existence of these dNTP imbalances in a proportion of retroviral permissive cells [13] has provided a mechanistic explanation for observed retroviral $G$ to $A$ hypermutations in vivo [11, 13-17]. These mis-incorporations result in polymorphisms, since reverse transcriptase lacks 3'-to-5' exonuclease proof-reading activity [18], provided that the mutations result in replication competent virus that is naturally selected in vivo. More recently, RNA editing, through APOBEC enzymes, that deaminate cytosine to uracil, have also been proposed as a mechanism for retroviral G to A hypermutation [19]. In fact, the newest role for the HIV-1 Vif protein, is to counter the effect of APOBEC [20, 21]. Interestingly, Vif may also be exploiting the mutation potential afforded by APOBEC, in generating more HIV-1 diversity [21], rather than simply providing immunity from the innate host APOBEC defense mechanism.

Importantly, both templated and non-templated miss-incorporations, as well as "slippage", can occur during the jumps of reverse transcription (Fig. 1) [22]. Therefore, in addition to point mutations, in vivo 
sampled retroviruses also frequently harbor deletions and insertions. Here, among the most probable mechanistic explanations, in addition to, or in combination with the above mentioned non-templated additions, is forced copy-choice strand switching during reverse transcription followed by mis-alignment and continued polymerization on the new template [23-28]. In essence this is a forced emulation of the reverse transcription "jumps" depicted in figure 1 [29]. This mechanism reconciles the fact that retroviruses can synthesize cDNA with only one RNA genomic copy [30] whereas viral particles carry 2 copies. The second would then serve the failsafe function of providing new template to "jump to" when reverse transcriptase encounters a nick. In addition, forced copy choice strand-switching provides a probable mechanistic explanation for phylogenetic revelations of inter-subtype recombinant HIV isolates [15, 31]. The implication is that chimeric exogenous retroviruses, containing transcribed genomic RNA from two different proviruses, are produced from doubly or multiply infected cells. In fact, recombination has now been shown to be the primary driving force for generating HIV-1 variants $[32,33]$, despite cellular retroviral superinfection resistance mechanisms [34].

The above retroviral mutation mechanisms highlight how retroviruses can generate sequence diversity. These mechanisms are shared by the larger family of elements capable of retro-transposition [7, 35]. However, HIV-1 undergoes such rapid exogenous viral replication and turnover [36-38], that natural selection generates enormously heterogeneous populations termed quasispecies $[16,39]$, or swarms, which have either temporally adapted to cell-specific microenvironments, or exist because their specific polymorphisms are neutral.

\section{The MFNLP}

The proviral HIV-1 LTR sequences first investigated, were from the initial isolate [40] and rapidly became the prototypical LTR. These sequences orchestrate the cellular RNA polymerase II transcription machinery, in conjunction with viral proteins, and include a TATA-box, 3 SP1 sites, 2 NFkB enhancer elements, an RBEIII site and an upstream USF site (Fig. 2A) (reviewed in [41]). Importantly, the extensive HIV-1 polymorphism detected in vivo, extends to the LTR $([15,42]$ and references therein).

In particular, a length polymorphism immediately $5^{\prime}$ of the enhancer region, has been detected, investigated, or referred to by a growing list of publications [42-60]. This polymorphism was initially detected as a 24 base pair duplication by Golub, Li and Volsky, looking for LTR variants; but no regulatory cis-acting motifs were described within it, despite a correlation with increased transcription [43]. Blumberg and co-authors, described a similar duplication, in adult and pediatric patients, while looking for variant nef sequences, but again did not find any motifs that might explain functional significance [44]. Koken and co-authors, were the first to refer to this duplication as the "CTG"-motif, and noted that it encompassed a repeat of the sequence 5'-ACTGCTGA-3', and further found that a single "CTG"-motif virus, could out-compete an isogenic virus containing the duplication, in co-culture experiments, in 42 days [45]. These authors also noted similarities between the duplicated region and the recently-described TCF-1 $\alpha$ (hLEF) binding site, and pointed out that HIVANT 70, as well as SIVCPZ, have the duplicated "CTG"-motif ([45] and references therein). These authors also pointed out that Nakanishi, Masamune and Kobayashi, had described the "CTG"-motif region as a novel cis-acting, cell-specific element [61]. In fact, in their paper, Nakanishi and co-authors designated this region (-157 to -121) the Upstream Regulatory Element (URE) and showed that it bound a transcription factor (designated URE-binding factors $\mathrm{C} 1$ and $\mathrm{C} 2$, by EMSA-specific analysis with nuclear extracts) distinct from AP-1 [61]. Furthermore, Koken and co-authors [45] noted that Zeichner, Kim and Alwine, had published data suggesting positive regulation in the "CTG"-motif region [62]. Soon afterwards, Kim, Gonzales, Zeichner and Alwine, published another paper, showing a more profound effect of mutations in the URE-region ("CTG"-motif), in the context of chromatin and viral replication, but attributed the effect to mutation of the TCF-1 $\alpha$ (hLEF) binding-site [63]. In another paper by Koken and co-authors, they extended their initial finding, first showing that "CTG"-motif deleted, and "CTG"-motif duplicated isogenic viruses, are out-competed in cell culture assays by the monomeric isomeric virus [46]. These authors also showed that a Circa $64 \mathrm{kD}$ protein, binds with specificity to the 5'-ACTGCTGA-3' sequence, duplicated in the "CTG"-motif duplications and obtained similar EMSA results to those of Nakanishi and co-authors, but further reported no correlation between the "CTG"-motif duplication and disease stage or viral phenotype $[46,61]$. So, by 1994 , it was already clear that a cis-element, named URE, enhanced viral replication, contained a putative binding site for TCF-1 $\alpha$ (hLEF), could specifically bind proteins from nuclear extracts, and was encompassed in a frequent Nef/LTR duplication ("CTG"-motif duplication). Furthermore it was clear that whatever the in vivo function of the "CTG"-motif duplication, in tissue culture assays with isogenic viruses, no dramatic effects were observed.

Further reports on this region of the HIV-1 LTR, included a paper from Michael and co-authors, that detected the TCF-1 $\alpha$ duplication in uncultured samples from patients, and suggested it may mediate a repressive effect [47], and a paper by Ait-Khaled, also detecting the TCF-1 $\alpha$ duplication, in post-mortem samples [48]. However, in 1996, in a cross-sectional analysis of nearly 500 HIV-1 LTRs, we published that the "CTG"-motif or URE region duplications, could best be aligned as a single larger polymorphism, which we named the most frequent naturally-occurring length polymorphism (MFNLP) of the HIV-1 LTR [42]. 
(A) Synthesis of minus strong stop DNA
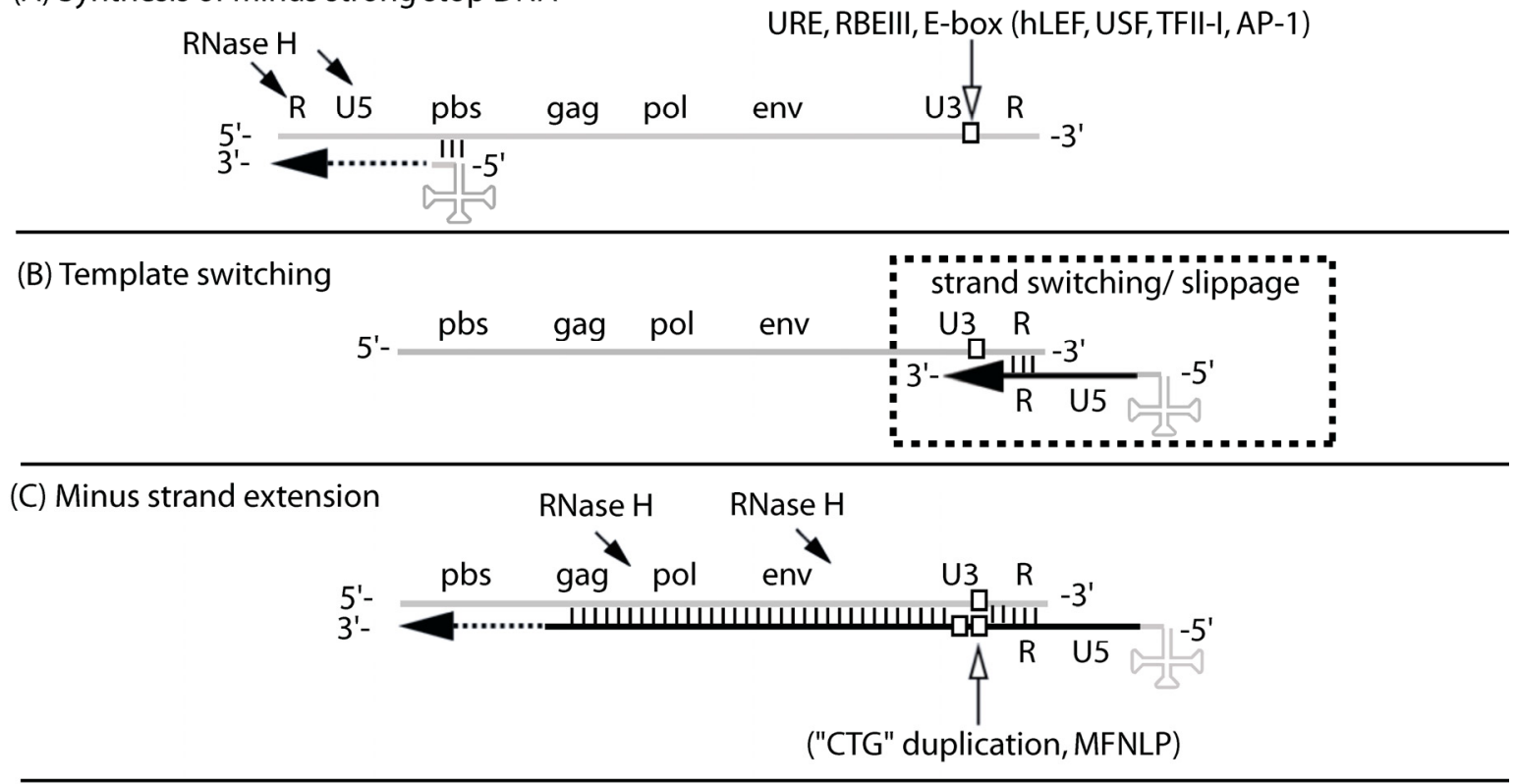

(D) Initial synthesis of plus strand

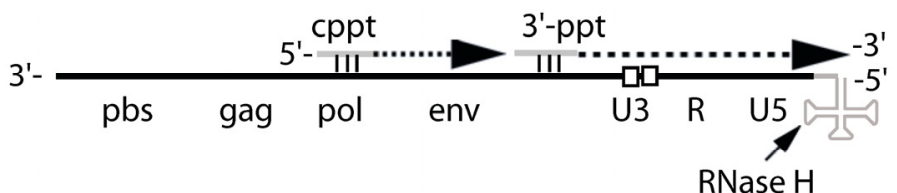

(E) Annealing/ extension/ nuclear import/ integration

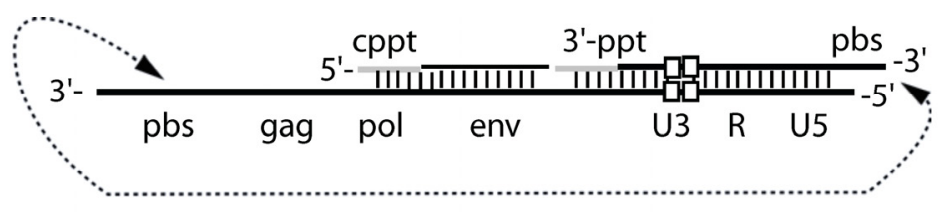

(F) Integrated HIV-1 provirus

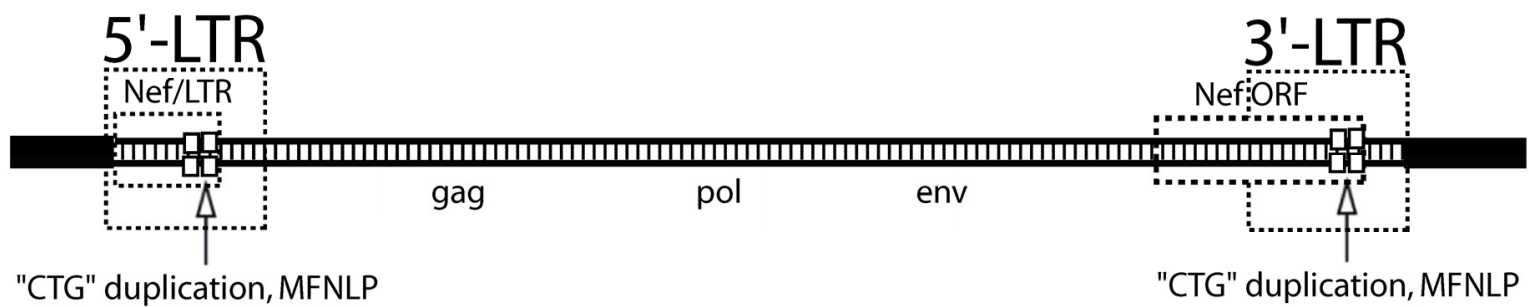

Figure 1. The MFNLP is generated during reverse transcription. (A) Synthesis of minus strong stop DNA, begins with t-RNA (clover-leaf structure) binding to the primer binding site (pbs), followed by RNA-templated polymerization of minus strong stop DNA (dashed arrow). RNase $\mathrm{H}$ degrades the template RNA after polymerization (arrows); (B) Template switching to the other RNA genome copy or to the same RNA, occurs via complementary sequences in the Repeat region (R). During the template switch, slippage can occur; (C) Minus strand extension could combine with slippage, templated and non-templated polymerization, resulting in duplication of the URE/RBEIII/partial E-box region (single white box, in RNA U3 region), generating a first copy of the MFNLP (two white boxes in U3 region), in the minus DNA. RNAse $\mathrm{H}$ continues to degrade the RNA genome, leaving just the polypurine tracts. (D) Initial synthesis of plus strand DNA begins from the central polypurine tract (cppt) and from the 3'-polypurine tract (3'-ppt), resulting in synthesis of a plus strand MFNLP (four white boxes); (E) Annealing of the free pbs, further extension and rearrangements, are followed by nuclear import and integration of the proviral HIV-1 sequences. (F) The final integrated proviral sequences contain a 5'-LTR and a 3'-LTR with U3, R, U5 sequences. In this model, both the 5' and 3'-LTR would contain the MFNLP. Note the Nef ORF in the 3'-LTR and the Nef/LTR sequences in the 5'-LTR. Integrity of the Nef TGA stop codon (ACTGCTGA) is critical for RBF-2 binding to the MFNLP. 
Furthermore, we proposed that the MFNLP encompassed a binding site for an unpublished nuclear factor named RBF-2, instead of TCF-1 $\alpha$ [42]. This transcription factor was defined in a subsequent publication [64], by a TGA-specificity for the 5'-ACTGCTGA-3' sequence, in crude nuclear extracts, and correlated with a region of the HIV-1 LTR required for optimal responsiveness to Ras signaling through the MAPK pathway, in tissue culture transient transfections. This sequence was named the Rasresponsive binding element III (RBEIII) [64]. Strikingly, a subsequent publication by Linqi Zhang and co-authors, reported a TCF-1 $\alpha$ duplication in a patient that progressed towards AIDS, despite the lack of $\mathrm{NF \kappa B}$ binding [49]. Interestingly, these authors suggested a compensatory role for the TCF- $1 \alpha$ duplication, in the face of NFKB binding-site abrogation, yet they found very little effect of the duplication in tissue culture [49]. Soon after, Kirchhoff and co-authors, reported on the presence of the MFNLP sequence in clinical samples, but did not point out that they duplicated RBEIII sequences, responsive to Ras [50]. Similarly, Zhang and co-authors, reported on more clinical samples with TCF-1 $\alpha$ duplications, for which they found no phenotype, but failed to point out that the MFNLPs duplicated RBEIII [51]. Quinones-Mateu, reported similar detection of sequence duplications, which we called MFNLPs [53]. In 1998, we further expanded our knowledge about the RBEIII and MFNLP-binding proteins, by showing: (i) that hLEF (TCF-1 $\alpha$ ) can footprint on the wild type site but not on all MFNLPs or RBEIII sites; (ii) that all MFNLPs bind RBF-2; and (iii) that a small repressive effect was mediated through the MFNLP in transient transfections [65]. In 1999 Naghavi and co-authors also reported TCF- $1 \alpha$ duplications in clinical samples $[55,66]$. At this time, we had already attempted to purify the components of RBF-2 by chromatography, including an oligo-affinity step [67]. Purified RBF-2 included proteins of approximately $120 \mathrm{kD}$ and $45 \mathrm{kD}$, however our repeated attempts to obtain peptide sequences from large scale purifications were inconclusive [67].

Importantly, in the lab of Robert Roeder, Naghavi and I began doing some experiments to confirm her sequencing data that predicted various HIV-1 subtypes would not bind to the NRE USF site, since the sequence was not highly conserved, as I had shown for samples from the long-standing Vancouver Lymphadenopathy Study (VLAS) cohort [42]. While studying the role of USF-1 in HIV-1 LTR-directed transcription and viral replication, we used a double stranded RBEIII site and a mutant RBEIII site, as a non-specific competitor in an EMSA with labeled double stranded E-box targets, encompassing the upstream USF-1 site in the prototypical LAV HIV-1 LTR sequence, and nuclear extracts (NRE B USF site, Fig 2B, lane1). Surprisingly, we found that at 100X molar excess, RBEIII competed with the band shift, but the RBEIII mutant did not (Fig 2B, lane 9 versus lane 10).
Although we at first assumed we had made a mistake, we immediately tested our new hypothesis that RBF2 contains USF-1, by competing a standard nuclear extract RBF2-RBEIII EMSA, with the E-box unlabelled target (Fig. 2C, lane 2). The hallmark of the RBF2 EMSA (Fig 2C, lane 1) is competition with 100X wild type RBEIII (Fig. 2C, lane 5) in the face of no competition with 100X mutant RBEIII (Fig. 2C, lane 4). The specific RBF2 bands were further tested with the non-specific 100X $w \mathrm{tNF} K \mathrm{~B}$ sequences from the prototype LAV HIV-1 LTR (Fig. 2C, lane 3). This important result indicated that our initial observation was true, and suggested that RBF2 contains USF-1. We therefore compared the EMSA mobility of rUSF-1 versus nuclear extracts, with an RBEIII target (Fig. 2D). The standard RBF-2 EMSA (Fig. 2D, lanes 1-4, lower arrow) appears to have a specific band that is lower in mobility than the specific band forming with rUSF-1 (Fig. $2 \mathrm{D}$, lanes 5-10). Importantly, although the mobility is slightly different, both bands are specific, since the nuclear extract band can be competed with 100X molar excess of wtRBEIII (lane 2) but not with 100X mut RBEIII (lane 3) and the rUSF-1 band can be competed with 100X wtRBEIII (lane 8) but not 100X mutRBEIII (lane 9). Additionally, antibodies to rUSF-1 appear to supershift both RBF-2 and rUSF-1 (lanes 4 and 10), consistent with USF-1 being in RBF-2. Since our focus is on the MFNLP and MFNLPs encompass RBEIII sequences, the above results strongly suggested that MFNLPs would also bind USF-1. However, the canonical E-box CANNTG site, recognized by USF-1, is not present in RBEIII sites. Indeed Target Detection Analysis (TDA), with random targets, using USF-1, does not select an RBEIII site [68]. However, we noted that in the purified RBF-2 preparations there was a circa $120 \mathrm{kD}$ component [67]. It was then Robert Roeder who immediately suggested to me that RBF-2 was comprised of USF and TFII-I. Because TFIIi and USF-1 bind the initiator/E-box region of the HIV-1 LTR, and RBF-2 had been shown to bind an RBEI site (in addition to the RBEIII site) that overlaps with the initiator sequences (Fig.2A) [64], the hypothesis that RBF-2 was comprised of USF and TFII-I seemed plausible. In fact, I found this was indeed the case, for at least 2 naturally occurring RBEIII HIV-1 LTR sequences (from clones pMCE36.1 and pMCE9.104, described elsewhere [65]), one of which is encompassed in an MFNLP (pMCE9.104) (Fig. 2D), in addition to the prototype HIV-1 LTR LAV sequences (above). Importantly, since binding of rUSF-1 alone (Fig. 2D, lanes 1 and 2) and binding of rTFIIi alone (lanes 5 and 6) is significantly weaker than when USF-1 is combined with TFIIi (lanes 3 and 4), it is likely that cooperative binding was occurring. Indeed, this important result suggested that USF-1 and TFIIi, when integrated at an RBEIII site, exert a stronger effect than individually, thus potentially explaining why their individual binding to an RBEIII site has not been previously observed. 
A
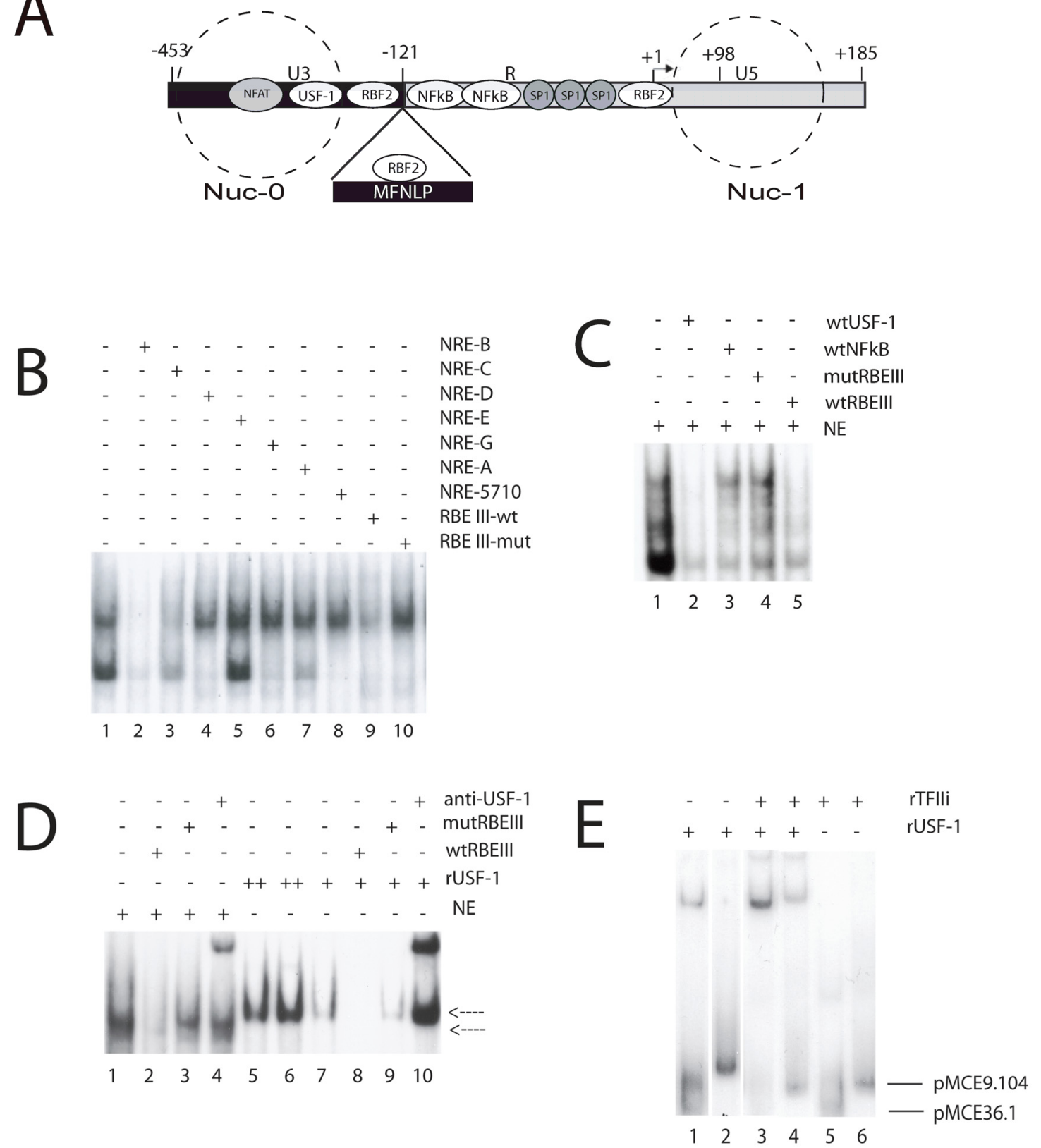

Figure 2. MFNLPs and RBEIII, bind USF-1 and TFIIi. (A) Schematic representation of the HIV-1 LTR. Some transcription factors landmarks (NFAT, USF-1, RBF2, NFkB, SP-1) known to interact with target sequences on the HIV-1 LTR are indicated in their relative positions. The -121 position where the MFNLPs occur is indicated, with the MFNLP above, bound by RBF2 at the MFNLP RBEIII site. The -453 and +185 sites indicate the extremes of the LTR. The +1 site indicates the start of transcription and the +98 site, the end of Tar sequences. The dashed circles represent the approximate positions of two nucleosomes (Nuc- 0 and Nuc-1). (B) RBEIII sequences specifically compete with a USF EMSA. The dsNREB oligosed as targets for the respective Hela nuclear extracts. Inclusion of competitor DNA sequences is indicated by a plus sign. (C) A USF-1 site competes specifically with RBF2 for an RBEIII site. A p32-labelled wt RBEIII ds oligo was used as target in EMSA with nuclear extracts from HeLa-s cells (NE, lanes 1-5). The target site that the respective competitor ds oligo sequences encompass, are designated with a + sign above each lane, and are listed on the right top of the panel. (D) rUSF-1 specifically binds to RBEIII, but results in a higher mobility EMSA than RBF2. A p32-labelled wt RBEIII ds oligo was used as target in EMSA with nuclear extracts from HeLa-s cells (NE, lanes 1-4) or with rUSF-1 (lanes 5-10). The target site that the respective competitor ds oligo sequences encompass, are designated with a + sign above each lane, and are listed on the right top of the panel. Lower arrow: NE-RBEIII (RBF2) complex. Upper arrow: rUSF-1-RBEIII complex. (D) rTFIIi and rUSF-1 bind cooperatively to MFNLP and wt RBEIII sites from 2 patients. A p32-labelled ds oligonucleotide target, encompassing the RBEIII site of either clone pMCE36.1 (lanes 1, 3, 5) or pMCE9.104 (lanes 2, 4, 6) was used as target in EMSA with either rUSF-1, TFIIi, or both, as indicated with a + sign above each lane, and listed at the right top of the panel. Lower arrow: Free pMCE36.1 ds oligonucleotide. Upper arrow: Free pMCE9.104 ds oligonucleotide. (B to E) The NRE, RBEIII, USF, NFKB sequences and EMSA conditions have been previously described [55, 64, 65, 67, 69]. 
Subsequent to our discovery, we asked the laboratory of Ivan Sadowski to confirm our results. Martin Hirst detected both USF and TFII-I in RBF-2 purifications [67], confirming our results. All the above initial findings were excluded from our publication about the NRE USF subtype sites [69], in order to better develop them into a clearer picture of the function of USF/TFII-I at the RBEIII sites, in collaboration with the lab of Ivan Sadowski (see below) [70].

Importantly, Jeeninga and co-authors, confirmed in 2000, that RBEIII sites are absolutely conserved, in LTR subtypes A through G [66]. A publication in 2000 by Gomez-Roman and co-authors, detected MFNLP sequences, duplicating RBEIII, in a long term non progressor (LTNP), but no deletions to RBEIII sites were found [56]. These LTNP are clinically distinct groups with lower viral replication and a slower or non-progressive form of HIV-1 infection (see below).

In 2000, Chen and co-authors found that an MFNLP from one of their patient samples, created a new AP-1 binding site, and identified c-Fos and JunB as binding proteins for their MFNLP [57]. Birch and co-authors described in 2001, LTNP from the Sydney Blood Bank Cohort (SBBC), that are accumulating progressively more LTR/Nef deletions, and some of these persons harbor RBEIII duplications in the form of MFNLPs [58]. Similarly, another LTNP study appears to have LTR/Nef sequences progressively accumulating greater deletions over time [60]. However it has not been at all clear if the LTR or the Nef deletions are responsible for the LTNP phenotype.

In 2002, Hiebenthal-Millow and Kirchhoff, found that in primary $\mathrm{T}$ cells, the MFNLP appears not to confer a major effect upon viral replication in culture [59]. A noteworthy observation about this paper is that these authors actually found enhanced transcriptional activity occurred when both the RBEIII site and MFNLPs were deleted, and that the MFNLP slightly reduced transcriptional activity.

Perhaps the most important paper about the role of the RBEIII site and the MFNLP is that from Chen and co-authors [70]. This paper expands on our initial discovery that RBF-2 is composed of TFIIi and USF, based on the TGA specificity of the EMSA used by Bell and Sadowski [64]. RBF-2 appears to be a heterodimer of USF-1/USF-2; and TFII-I appears to load USF-1/ USF-2 onto the RBEIII site. Importantly, this paper shows that RBEIII mutant LTRs are unresponsive to cross-linking of the $\mathrm{T}$ cell receptor or stimulation with PMA and ionomycin. Thus it appears that the phenotype of the RBEIII site and hence the compensatory MFNLP role, is to provide a target for Ras/MAPK activation of the HIV-1 LTR, in the context of chromatin [70, 71].

\section{Nef, LTNP and RBF-2}

Importantly, the most recent SBBC LTNP study, indicates that there is a convergence in LTR/Nef deletions for these LTNPs [72]. These deletions appear to leave the sequences downstream of the NFKB sites intact, yet appear to be abrogating RBEIII sites, since they are deleted, without MFNLPs. Importantly, there are publications indicating that Nef can activate the MAPK pathway [73] and increase T cell activation [74]. Therefore Nef could be actually acting through the Nef/LTR cis-acting RBEIII site, which is abrogated in the SSBC LTNPs [72]. I therefore propose the model in figure 3. LTRs with normal RBEIII sites and a normal Nef sequence, could contribute to high viral replication and progress to AIDS (Fig 3A). The presence of MFNLPs in the context of normal RBEIII sites would simply provide a failsafe function. However, LTRs with mutated RBEIII site would require an MFNLP, as a compensatory mechanism (Fig 3 B). If however, LTRs receive difficult-to-revert Nef deletions, either after an RBEIII mutation (Fig. 3C) or prior to an RBEIII mutation (Fig. 3D), then the selective pressure to maintain the RBEIII site and the MFNLP disappears. The result of this model, would explain the convergent deletions observed in the LTR/Nef SSBC LTNP study, as well as being consistent with the role for RBF-2, RBEIII and MFNLPs.

Whether my model about LTNP is true or not, the conserved nature of the RBEIII sites in vivo, makes it an important potential target for delivery of chimeric transcription factor repressors, which could emulate the LTNP phenotype, by blocking activation through this highly conserved site [75]. This is illustrated in Figure 4, where a chimeric transcription factor encompassing a repressor (such as MCEF[75, 76]) and a DNA binding partner (RBF-2), might have the potential to both block tat-transactivation and interfere with the function of RBEIII.

\section{Competing interests}

I have no competing interests.

\section{Acknowledgements}

I thank Mojgan Naghavi for providing her data (Fig. 2D), Robert Roeder for use of his lab and reagents between 1998 and 2001, Ivan Sadowski for the use of his lab and reagents between 1993 and 1998, and Martin Hirst for his role in confirming our RBF-2 preparations in the Sadowski lab contained USF and TFII-I. Maksymillian Niedzielski helped with making the figures and Robert Hopewell helped with the bibliography. The writing of this review was supported in part by grants from Canfar, CFI, OIT, NSERC and CIHR. 
(A) MFNLP Failsafe Function

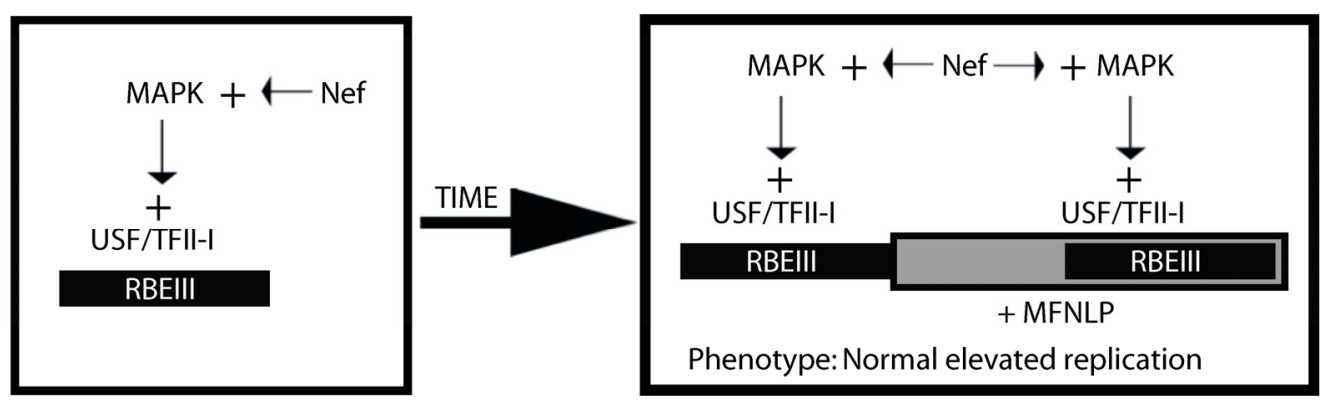

(B) MFNLP Compensatory Function

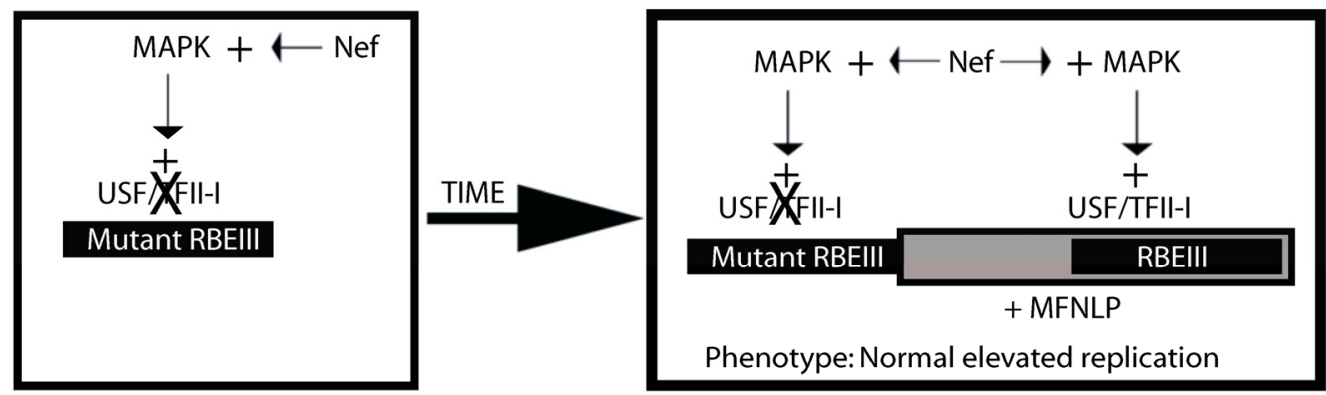

(C) Difficult to Revert Nef/LTR Deletions

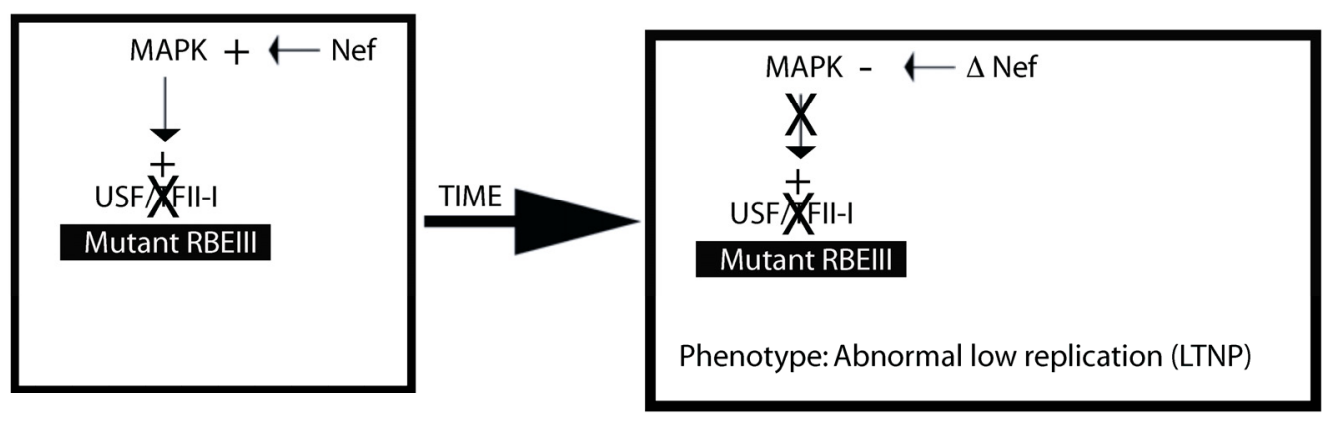

\section{(D) Difficult to Revert Nef/LTR Deletions}

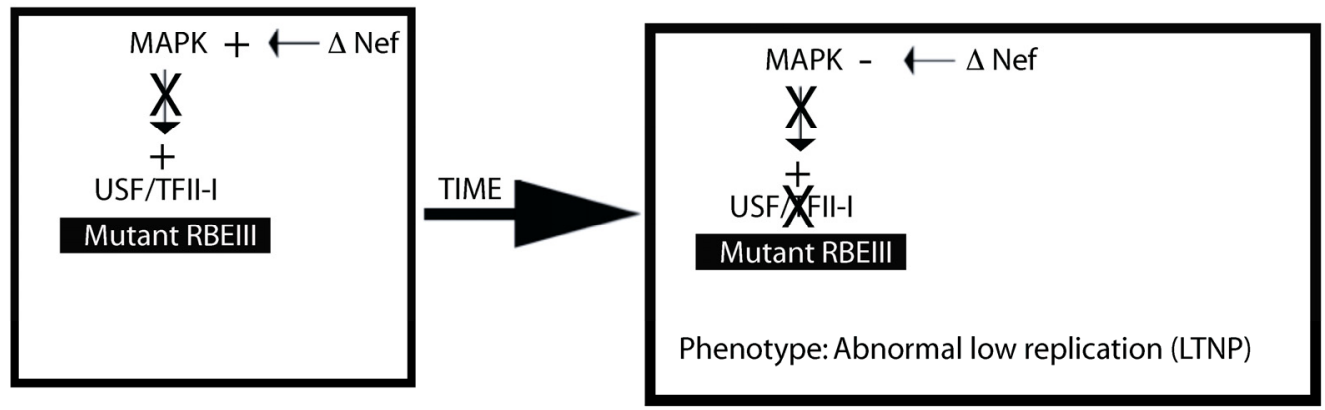

Figure 3. MFNLPs compensate for RBEIII mutations, by maintaining functional coupling, of Nef and RBF-2. In this schematic representation four states are considered. In A \& B, normal progressive disease with high viral titer would develop. In C \& $\mathrm{D}$, difficult-to-revert-mutations to Nef or the RBEIII site, would result in LTNP, and deletions of both RBEIII LTR sequences and Nef sequences. This is because, in our model, Nef can activate the MAPK pathway, and exert its effects through RBEIII. 


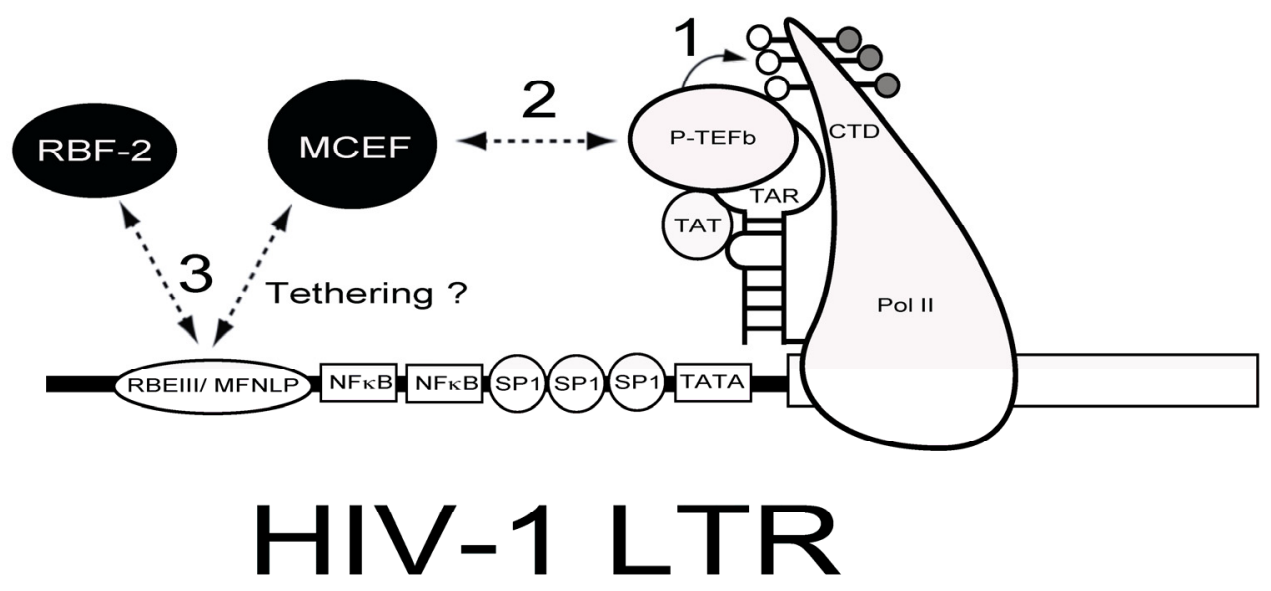

Figure 4. Chimeric Transcription Factor Repression (CTFR) of HIV-1. Delivery of a repressor with the ability to interfere with Tat-transactivation and bind to the RBEIII/ MFNLP is hypothesized. The HIV-1 LTR is drawn with some salient transcription factors indicated. The RNA stem-loop structure termed TAR, can form a ternary complex with HIV-1 TA and P-TEFb, to hyperphosphorylate the carboxy terminal domain (CTD) of RNA Polymerase II (PolII). Because MCEF interacts with P-TEFb and represses HIV-1 Tat-transactivation, if tethered to the RBEIII/ MFNLP site, MCEF could interfere with the binding of RBF-2 (3) and tat transactivation (1 and 2).

\section{References}

1. Barre-Sinoussi F, Chermann JC, Rey F, Nugeyre MT, Chamaret S, Gruest J, Dauguet C, Axler-Blin C, Vezinet-Brun F, Rouzioux C, Rozenbaum W, and Montagnier L. Isolation of a T-lymphotropic retrovirus from a patient at risk for acquired immune deficiency syndrome (AIDS). Science 1983; 220: 868-871

2. Wattel E, Vartanian JP, Pannetier C, and Wain-Hobson S. Clonal expansion of human T-cell leukemia virus type I-infected cells in asymptomatic and symptomatic carriers without malignancy. J Virol 1995; 69: 2863-2868

3. Gessain A, Gallo RC, and Franchini G. Low degree of human T-cell leukemia/lymphoma virus type I genetic drift in vivo as a means of monitoring viral transmission and movement of ancient human populations. J Virol 1992; 66: 2288-2295

4. Wain-Hobson S. Running the gamut of retroviral variation. Trends Microbiol 1996; 4: 135-141

5. Komurian F, Pelloquin F, and de The G. In vivo genomic variability of human T-cell leukemia virus type I depends more upon geography than upon pathologies. J Virol 1991; 65: 3770-3778

6. Kunkel TA. DNA replication fidelity. J Biol Chem 1992; 267: 18251-18254

7. Preston $\mathrm{BD}$, and Dougherty JP. Mechanisms of retroviral mutation. Trends Microbiol 1996; 4: 16-21

8. Zenkin N, Yuzenkova Y, and Severinov K. Transcript-assisted transcriptional proofreading. Science 2006; 313: 518-520

9. Freed EO. HIV-1 replication. Somat Cell Mol Genet 2001; 26: 13-33

10. Rausch JW, and Le Grice SF. 'Binding, bending and bonding': polypurine tract-primed initiation of plus-strand DNA synthesis in human immunodeficiency virus. Int J Biochem Cell Biol 2004; 36: $1752-1766$

11. Cheynier R, Gratton S, Vartanian JP, Meyerhans A, and Wain-Hobson S. G --> A hypermutation does not result from polymerase chain reaction. AIDS Res Hum Retroviruses 1997; 13 985-986

12. Meyerhans A, Vartanian JP, Hultgren C, Plikat U, Karlsson A, Wang L, Eriksson S, and Wain-Hobson S. Restriction and enhancement of human immunodeficiency virus type 1 replication by modulation of intracellular deoxynucleoside triphosphate pools. J Virol 1994; 68: 535-540

13. Vartanian JP, Plikat U, Henry M, Mahieux R, Guillemot L, Mey- erhans A, and Wain-Hobson S. HIV genetic variation is directed and restricted by DNA precursor availability. J Mol Biol 1997; 270: 139-151

14. Vartanian JP, Meyerhans A, Sala M, and Wain-Hobson S. G-->A hypermutation of the human immunodeficiency virus type 1 genome: evidence for dCTP pool imbalance during reverse transcription. Proc Natl Acad Sci U S A 1994; 91: 3092-3096

15. Delassus S, Cheynier R, and Wain-Hobson S. Evolution of human immunodeficiency virus type 1 nef and long terminal repeat sequences over 4 years in vivo and in vitro. J Virol 1991; 65: 225-231

16. Goodenow M, Huet T, Saurin W, Kwok S, Sninsky J, and Wain-Hobson S. HIV-1 isolates are rapidly evolving quasispecies: evidence for viral mixtures and preferred nucleotide substitutions. J Acquir Immune Defic Syndr 1989; 2: 344-352

17. Vartanian JP, Meyerhans A, Asjo B, and Wain-Hobson S. Selection, recombination, and G----A hypermutation of human immunodeficiency virus type 1 genomes. J Virol 1991; 65: 1779-1788

18. Skalka A, and Goff S. Reverse Transcriptase. New York: Cold Spring Harbor Press. 1993

19. Vartanian JP, Sommer P, and Wain-Hobson S. Death and the retrovirus. Trends Mol Med 2003; 9: 409-413

20. Suspene R, Rusniok C, Vartanian JP, and Wain-Hobson S. Twin gradients in APOBEC3 edited HIV-1 DNA reflect the dynamics of lentiviral replication. Nucleic Acids Res 2006; 34: 4677-4684

21. Simon V, Zennou V, Murray D, Huang Y, Ho DD, and Bieniasz PD. Natural variation in Vif: differential impact on APOBEC 3G/3F and a potential role in HIV-1 diversification. PLoS Pathog 2005; 1: e6

22. Patel PH, and Preston BD. Marked infidelity of human immunodeficiency virus type 1 reverse transcriptase at RNA and DNA template ends. Proc Natl Acad Sci U S A 1994; 91: 549-553

23. Allain B, Lapadat-Tapolsky M, Berlioz C, and Darlix JL. Transactivation of the minus-strand DNA transfer by nucleocapsid protein during reverse transcription of the retroviral genome. Embo J 1994; 13: 973-981

24. Darlix JL, Vincent A, Gabus C, de Rocquigny H, and Roques B. Trans-activation of the $5^{\prime}$ to $3^{\prime}$ viral DNA strand transfer by nucleocapsid protein during reverse transcription of HIV1 RNA. C R Acad Sci III 1993; 316: 763-771

25. Guo J, Henderson LE, Bess J, Kane B, and Levin JG. Human immunodeficiency virus type 1 nucleocapsid protein promotes efficient strand transfer and specific viral DNA synthesis by inhibiting TAR-dependent self-priming from minus-strand 
strong-stop DNA. J Virol 1997; 71: 5178-5188

26. Klaver B, and Berkhout B. Comparison of $5^{\prime}$ and $3^{\prime}$ long terminal repeat promoter function in human immunodeficiency virus. J Virol 1994; 68: 3830-3840

27. Ramsey CA, and Panganiban AT. Replication of the retroviral terminal repeat sequence during in vivo reverse transcription. J Virol 1993; 67: 4114-4121

28. Temin HM. Retrovirus variation and reverse transcription: abnormal strand transfers result in retrovirus genetic variation. Proc Natl Acad Sci U S A 1993; 90: 6900-6903

29. Panganiban AT, and Fiore D. Ordered interstrand and intrastrand DNA transfer during reverse transcription. Science 1988; 241: 1064-1069

30. Jones JS, Allan RW, and Temin HM. One retroviral RNA is sufficient for synthesis of viral DNA. J Virol 1994; 68: 207-216

31. Gao F, Robertson DL, Morrison SG, Hui H, Craig S, Decker J, Fultz PN, Girard M, Shaw GM, Hahn BH, and Sharp PM. The heterosexual human immunodeficiency virus type 1 epidemic in Thailand is caused by an intersubtype (A/E) recombinant of African origin. J Virol 1996; 70: 7013-7029

32. Jung A, Maier R, Vartanian JP, Bocharov G, Jung V, Fischer U, Meese E, Wain-Hobson S, and Meyerhans A. Multiply infected spleen cells in HIV patients. Nature 2002; 418: 144

33. Meyerhans A, Jung A, Maier R, Vartanian JP, Bocharov G, and Wain-Hobson S. The non-clonal and transitory nature of HIV in vivo. Swiss Med Wkly 2003; 133: 451-454

34. Nethe M, Berkhout B, and van der Kuyl AC. Retroviral superinfection resistance. Retrovirology 2005; $2: 52$

35. Preston BD. Error-prone retrotransposition: rime of the ancient mutators. Proc Natl Acad Sci U S A 1996; 93: 7427-7431

36. Coffin JM. HIV population dynamics in vivo: implications for genetic variation, pathogenesis, and therapy. Science 1995; 267: 483-489

37. Ho DD, Neumann AU, Perelson AS, Chen W, Leonard JM, and Markowitz M. Rapid turnover of plasma virions and CD4 lymphocytes in HIV-1 infection. Nature 1995; 373: 123-126

38. Wei X, Ghosh SK, Taylor ME, Johnson VA, Emini EA, Deutsch P, Lifson JD, Bonhoeffer S, Nowak MA, Hahn BH, et al.. Viral dynamics in human immunodeficiency virus type 1 infection. Nature 1995; 373: 117-122

39. Biebricher CK, and Eigen M. What is a quasispecies? Curr Top Microbiol Immunol 2006; 299: 1-31

40. Wain-Hobson S, Sonigo P, Danos O, Cole S, and Alizon M. Nucleotide sequence of the AIDS virus, LAV. Cell 1985; 40: 9-17

41. Pereira LA, Bentley K, Peeters A, Churchill MJ, and Deacon NJ. A compilation of cellular transcription factor interactions with the HIV-1 LTR promoter. Nucleic Acids Res 2000; 28: 663-668

42. Estable MC, Bell B, Merzouki A, Montaner JS, O'Shaughnessy MV, and Sadowski IJ. Human immunodeficiency virus type 1 long terminal repeat variants from 42 patients representing all stages of infection display a wide range of sequence polymorphism and transcription activity. J Virol 1996; 70: 4053-4062

43. Golub E, Li G, and Volsky D. Differences in the basal activity of the long terminal repeat determine different replicative capacities of two closely related human immunodeficiency virus type 1 isolates. J Virol. 1991; 64: 3654-3660

44. Blumberg BM, Epstein LG, Saito Y, Chen D, Sharer LR, and Anand R. Human immunodeficiency virus type 1 nef quasispecies in pathological tissue. J Virol 1992; 66: 5256-5264

45. Koken SE, van Wamel JL, Goudsmit J, Berkhout B, and Geelen JL. Natural variants of the HIV-1 long terminal repeat: analysis of promoters with duplicated DNA regulatory motifs. Virology 1992; 191: 968-972

46. Koken SE, van Wamel JL, Geelen JL, and Berkhout B. Functional Analysis of the ACTGCTGA Sequence Motif in the Human Immunodeficiency Virus Type-1 Long Terminal Repeat Promoter. J Biomed Sci 1994; 1: 83-92
47. Michael NL, D'Arcy L, Ehrenberg PK, and Redfield RR. Naturally occurring genotypes of the human immunodeficiency virus type 1 long terminal repeat display a wide range of basal and Tat-induced transcriptional activities. J Virol 1994; 68: 3163-3174

48. Ait-Khaled M, McLaughlin JE, Johnson MA, and Emery VC. Distinct HIV-1 long terminal repeat quasispecies present in nervous tissues compared to that in lung, blood and lymphoid tissues of an AIDS patient. Aids 1995; 9: 675-683

49. Zhang L, Huang Y, Yuan H, Chen BK, Ip J, and Ho DD. Identification of a replication-competent pathogenic human immunodeficiency virus type 1 with a duplication in the TCF-1alpha region but lacking NF-kappaB binding sites. J Virol 1997; 71: 1651-1656

50. Kirchhoff F, Greenough TC, Hamacher M, Sullivan JL, and Desrosiers RC. Activity of human immunodeficiency virus type 1 promoter/TAR regions and tat 1 genes derived from individuals with different rates of disease progression. Virology 1997; 232: 319-331

51. Zhang L, Huang Y, Yuan H, Chen BK, Ip J, and Ho DD. Genotypic and phenotypic characterization of long terminal repeat sequences from long-term survivors of human immunodeficiency virus type 1 infection. J Virol 1997; 71: 5608-5613

52. Salvi R, Garbuglia AR, Di Caro A, Pulciani S, Montella F, and Benedetto A. Grossly defective nef gene sequences in a human immunodeficiency virus type 1-seropositive long-term nonprogressor. J Virol 1998; 72: 3646-3657

53. Quinones-Mateu ME, Mas A, Lain de Lera T, Soriano V, Alcami J, Lederman MM, and Domingo E. LTR and tat variability of HIV-1 isolates from patients with divergent rates of disease progression. Virus Res 1998; 57: 11-20

54. Estable MC, Merzouki A, Arella M, and Sadowski IJ. Distinct clustering of HIV type 1 sequences derived from injection versus noninjection drug users in Vancouver, Canada. AIDS Res Hum Retroviruses 1998; 14: 917-919

55. Naghavi MH, Schwartz S, Sonnerborg A, and Vahlne A. Long terminal repeat promoter/enhancer activity of different subtypes of HIV type 1. AIDS Res Hum Retroviruses 1999; 15: 1293-1303

56. Gomez-Roman VR, Vazquez JA, et al. nef/long terminal repeat quasispecies from HIV type 1-infected Mexican patients with different progression patterns and their pathogenesis in hu-PBL-SCID mice. AIDS Res Hum Retroviruses 2000; 16: 441-452

57. Chen P, Flory E, Avots A, Jordan BW, Kirchhoff F, Ludwig S, and Rapp UR. Transactivation of naturally occurring HIV-1 long terminal repeats by the JNK signaling pathway. The most frequent naturally occurring length polymorphism sequence introduces a novel binding site for AP-1 factors. J Biol Chem 2000; 275: 20382-20390

58. Birch MR, Learmont JC, Dyer WB, Deacon NJ, Zaunders JJ, Saksena N, Cunningham AL, Mills J, and Sullivan JS. An examination of signs of disease progression in survivors of the Sydney Blood Bank Cohort (SBBC). J Clin Virol 2001; 22: 263-270

59. Hiebenthal-Millow K, and Kirchhoff F. The most frequent naturally occurring length polymorphism in the HIV-1 LTR has little effect on proviral transcription and viral replication. Virology 2002; 292: 169-175

60. Kondo M, Shima T, Nishizawa M, Sudo K, Iwamuro S, Okabe T, Takebe $\mathrm{Y}$, and Imai M. Identification of attenuated variants of HIV-1 circulating recombinant form 01_AE that are associated with slow disease progression due to gross genetic alterations in the nef/long terminal repeat sequences. J Infect Dis 2005; 192: 56-61

61. Nakanishi Y, Masamune Y, and Kobayashi N. A novel cis-acting element that controls transcription of human immunodeficiency virus type 1 DNA, depending on cell type. J Virol 1991; 65: 6334-6338 
62. Zeichner SL, Kim JY, and Alwine JC. Linker-scanning mutational analysis of the transcriptional activity of the human immunodeficiency virus type 1 long terminal repeat. J Virol 1991; 65: 2436-2444

63. Kim JY, Gonzalez-Scarano F, Zeichner SL, and Alwine JC. Replication of type 1 human immunodeficiency viruses containing linker substitution mutations in the -201 to -130 region of the long terminal repeat. J Virol 1993; 67: 1658-1662

64. Bell B, and Sadowski I. Ras-responsiveness of the HIV-1 LTR requires RBF-1 and RBF-2 binding sites. Oncogene 1996; 13: 2687-2697

65. Estable MC, Bell B, Hirst M, and Sadowski I. Naturally occurring human immunodeficiency virus type 1 long terminal repeats have a frequently observed duplication that binds RBF-2 and represses transcription. J Virol 1998; 72: 6465-6474

66. Jeeninga RE, Hoogenkamp M, Armand-Ugon M, de Baar M, Verhoef $\mathrm{K}$, and Berkhout B. Functional differences between the long terminal repeat transcriptional promoters of human immunodeficiency virus type 1 subtypes A through G. J Virol 2000; 74: 3740-3751

67. Estable MC, Hirst M, Bell B, O'Shaughnessy MV, and Sadowski I. Purification of RBF-2, a transcription factor with specificity for the most conserved cis-element of naturally occurring HIV-1 LTRs. J Biomed Sci 1999; 6: 320-332

68. Bendall AJ, and Molloy PL. Base preferences for DNA binding by the bHLH-Zip protein USF: effects of $\mathrm{MgCl} 2$ on specificity and comparison with binding of Myc family members. Nucleic Acids Res 1994; 22: 2801-2810

69. Naghavi MH, Estable MC, Schwartz S, Roeder RG, and Vahlne A. Upstream stimulating factor affects human immunodeficiency virus type 1 (HIV-1) long terminal repeat-directed transcription in a cell-specific manner, independently of the HIV-1 subtype and the core-negative regulatory element. J Gen Virol 2001; 82: 547-559

70. Chen J, Malcolm T, Estable MC, Roeder RG, and Sadowski I. TFII-I regulates induction of chromosomally integrated human immunodeficiency virus type 1 long terminal repeat in cooperation with USF. J Virol 2005; 79: 4396-4406

71. Sadowski I, and Mitchell DA. TFII-I and USF (RBF-2) regulate Ras/MAPK-responsive HIV-1 transcription in T cells. Eur J Cancer 2005; 41: 2528-2536

72. Churchill MJ, Rhodes DI, Learmont JC, Sullivan JS, Wesselingh SL, Cooke IR, Deacon NJ, and Gorry PR. Longitudinal analysis of human immunodeficiency virus type 1 nef/long terminal repeat sequences in a cohort of long-term survivors infected from a single source. J Virol 2006; 80: 1047-1052

73. Schrager JA, Der Minassian V, and Marsh JW. HIV Nef increases T cell ERK MAP kinase activity. J Biol Chem 2002; 277: 6137-6142

74. Schrager JA, and Marsh JW. HIV-1 Nef increases T cell activation in a stimulus-dependent manner. Proc Natl Acad Sci U S A 1999; 96: 8167-8172

75. Niedzielski MF, Hopewell R, Ismail Z, and Estable MC. MCEF is localized to the nucleus by protein sequences encoded within three distinct exons, where it represses HIV-1 Tat-transactivation of LTR-directed transcription. Int J Biol Sci 2007; 3: 225-236

76. Estable MC, Naghavi MH, Kato H, Xiao H, Qin J, Vahlne A, and Roeder RG. MCEF, the newest member of the AF4 family of transcription factors involved in leukemia, is a positive transcription elongation factor-b-associated protein. J Biomed Sci 2002; 9: 234-245 